\title{
Plasma functionalization of polycarbonaturethane to improve endothelialization-Effect of shear stress as a critical factor for biocompatibility control
}

Journal of Biomaterials Applications 2016, Vol. 30(9) 1417-1428 (C) The Author(s) 2016 Reprints and permissions: sagepub.co.uk/journalsPermissions.nav DOI: $10.1177 / 0885328215626072$ jba.sagepub.com

@SAGE

\author{
Karin Lukas', Ulrich Thomas², André Gessner', \\ Daniel Wehner ${ }^{2}$, Thomas Schmid ${ }^{2}$, Christof Schmid ${ }^{3}$ and \\ Karla Lehle ${ }^{3}$
}

\begin{abstract}
Medical devices made of polycarbonaturethane (PCU) combine excellent mechanical properties and little biological degradation, but restricted hemocompatibility. Modifications of PCU might reduce platelet adhesion and promote stable endothelialization. PCU was modified using gas plasma treatment, binding of hydrogels, and coupling of cellactive molecules (modified heparin, anti-thrombin III (ATIII), argatroban, fibronectin, laminin-nonapeptide, peptides with integrin-binding arginine-glycine-aspartic acid (RGD) motif). Biocompatibility was verified with static and dynamic cell culture techniques. Blinded analysis focused on improvement in endothelial cell (EC) adhesion/proliferation, antithrombogenicity, reproducible manufacturing process, and shear stress tolerance of ECs. EC adhesion and antithrombogenicity were achieved with 9/35 modifications. Additionally, 6/9 stimulated EC proliferation and 3/6 modification processes were highly reproducible for endothelialization. The latter modifications comprised immobilization of ATIII $(A)$, polyethyleneglycole-diamine-hydrogel $(E)$ and polyethylenimine-hydrogel connected with modified heparin $(\mathrm{IH})$. Under sheer stress, only the IH modification improved EC adhesion within the graft. However, ECs did not arrange in flow direction and cell anchorage was restricted. Despite large variation in surface modification chemistry and improved EC adhesion under static culture conditions, additional introduction of shear stress foiled promising preliminary data. Therefore, biocompatibility testing required not only static tests but also usage of physiological conditions such as shear stress in the case of vascular grafts.
\end{abstract}

\section{Keywords}

Endothelial cell seeding, thrombogenicity, plasma treatment, hydrogel, polyurethane, sheer stress, biocompatibility

\section{Introduction}

Polycarbonateurethanes (PCUs) have been used for producing biostable implantable medical devices (e.g. artificial blood vessels, small-diameter vascular grafts) as they provide relatively beneficial thromboresistance, biocompatibility and mechanical properties similar to the polyether-based polyurethanes. ${ }^{1-4}$ However, thrombus formation remained a critical risk factor. ${ }^{5-7} \mathrm{An}$ effective way to improve hemocompatibility of PCU is to optimize its material surface but not significantly change their intrinsic mechanical properties. Modification of the polyurethane surface chemistry
'IMHR, Institute for Medical Microbiology and Hygiene, University of Regensburg, Regensburg, Germany

${ }^{2}$ Dualis Medtech GmbH, Seefeld, Germany

${ }^{3}$ Department of Cardiothoracic Surgery, University Hospital Regensburg, Regensburg, Germany

Corresponding author:

Karla Lehle, Department of Cardiothoracic Surgery, University Hospital Regensburg, Franz-Josef-Strauss-Allee II, D-93042 Regensburg,

Germany.

Email: Karla.Lehle@UKR.de 
combining reduced platelet adhesion/activation and increasing endothelial attachment would be an attractive task.

It is very attractive to activate polymer surfaces using gas plasma treatment. ${ }^{8-13}$ In this context, it was shown that nitrogen $\left(\mathrm{N}_{2}\right)$ plasma treatment enriched the surface with nitrogen species,, 10 and thereby reduced platelet adhesion, activation, and aggregation. ${ }^{9,11}$ In addition, $\mathrm{N}_{2}$ plasma activation promoted adhesion and proliferation of fibroblasts and osteoblasts onto chitosan membranes or biodegradable polybutylene succinate surfaces. ${ }^{12,13}$ Furthermore, biocompatible hydrogels are generating growing interest in the community of tissue engineering because of their mechanical and chemical versatility and cell compatibility based on biomimicry of the extracellular matrix. ${ }^{14,15}$ For example, polyethylene glycol (PEG) is one of the most widely investigated hydrogels, ${ }^{16}$ that allowed adhesion of smooth muscle cells for improvement of cardiovascular tissue engineering applications. ${ }^{17}$ Furthermore, PEG-diamines were used as branch molecules in the network which were linked to cell-active molecules. ${ }^{18}$ Other interesting hydrogels in tissue engineering approaches are polyethyleneimine composites $^{19,20}$ and carboxymethyl dextran ${ }^{21}$. They are characterized by low cytotoxicity, simple processing, and variable applicability. ${ }^{19}$ Additional immobilization of biomolecules on biomaterial surfaces has been also proven to be a very effective method for considerable improvement of blood compatibility or increasing cell attachment and proliferation. ${ }^{22}$ Covalent binding of fibronectin ${ }^{23}$ or RGD peptides ${ }^{24,25}$ as well as a laminin-nonapeptide ${ }^{26}$ improved integrin-mediated cell adhesion of endothelial cells or progenitor cells. Other strategies such as binding of anticoagulants like heparin, ${ }^{27}$ phosphorylcholine, ${ }^{28}$ or Argatroban ${ }^{29}$ pursued the aim to reduce platelet adhesion, activation and aggregation.

Routinely, biocompatibility testing only used static culture methods according to international standard ISO-10993 requirements. ${ }^{8,11,12,22-25,28}$ In cardiovascular tissue engineering, endothelial cell attachment to and retention on an appropriate scaffold is particularly important because of the high fluid shear forces applied to the graft lumen upon implantation within the arterial tree. Therefore, bioreactors and perfusion flow systems were used to endothelialize the graft lumen, to precondition the cells to blood flow and to mimic the physiological fluid-induced cyclical shear stress derived from blood flow through the vessels. ${ }^{14,22,30}$ In the present study, static methods were introduced to analyze the bio- and hemocompatibiltiy of 35 different chemical modifications of PCU. The most promising modifications with low platelet adhesion and improved endothelial cell adhesion were analyzed in a bioreactor under pulsatile flow to evaluate the effect of shear stress on the integrity of the cell monolayer.

\section{Materials and methods}

\section{Materials}

Disks (area, $0.3 \mathrm{~cm}^{2}$ ) and vascular grafts (length, $15 \mathrm{~cm}$; diameter, $0.6 \mathrm{~cm}$; wall thickness, $0.5 \mathrm{~mm}$ ) made of PCU were purchased from DUALIS (Seefeld, Germany). The PCU-samples were cleaned (ethanol, p.a.) and air dried before processing.

$\mathrm{N}_{2}$ and carbon dioxide $\left(\mathrm{CO}_{2}\right)$ were purchased from Linde (ultrapure grade, 99.998\%; Ludwigshafen, Germany). Laminin-nonapeptide (CDPGYJGSR), short (GRGDSPK) and long (GCGYGRGDSPG) RGD-peptides were obtained from GenScript Corporation (Piscataway, NJ), Antithrombin III (ATIII, Kybernin ${ }^{\circledR} \mathrm{P}$ ) from CLS Behring (Marburg, Germany), Argatroban (Argatra ${ }^{\circledR}$ ) from Mitsubishi Pharma (Düsseldorf, Germany), human fibronectin from BD Biosciences (Heidelberg, Germany), branched polyethyleneimine (PEI) and poly(ethylene glycol) bis(amine), (PEG-diamine, MW10,000) from Sigma-Aldrich (Munich, Germany). Unless otherwise indicated, chemicals were purchased from Sigma-Aldrich or Merck (Darmstadt, Germany). Water was deionized. Carbonate buffer (c-buffer) $(0.1 \mathrm{M}, \mathrm{pH} 8.4)$ was prepared freshly. Carboxymethyl-dextran (CM-Dex) was prepared with $1 \mathrm{M}$ bromoacetic acid as described earlier. ${ }^{31}$ Heparin was modified by dissolving heparin $(0.3 \%)$ in icy water, spiked with sodium nitrite $(0.4 \mathrm{M})$, acidified with hydrogen chloride $(\mathrm{pH} 2.7)$, and stirred $\left(2 \mathrm{~h}, 0^{\circ} \mathrm{C}\right)$. After adjustment to $\mathrm{pH} 7.0$, the solution was filled with twice the volume of $2 \%$ sodium chloride $\left(\mathrm{NaCl}\right.$, in ethanol) and stored for $40 \mathrm{~h}\left(4^{\circ} \mathrm{C}\right)$. Crystals were harvested, dried in vacuum and stored at $4{ }^{\circ} \mathrm{C}$.

\section{Surface modifications}

Figure 1 presents all analyzed surface modifications using different manufacturing processes (Argatroban, Ar; ATIII, A; fibrinogen, F; Laminin-nonapeptide, L; short, Rs, and long, R1, RGD-peptides). Due to blinded study design and block by block biological testing, not all molecules were immobilized onto each activated surface.

Procedure I. All PCU-samples were plasma treated ${ }^{32}$ using a Plasmabrush ${ }^{\circledR}$ (Reinhausen Plasma, Germany). The plasma chamber was thoroughly purged with a continuous flow of $\mathrm{N}_{2}(20 \mathrm{NL} / \mathrm{min}$; voltage, $4500 \mathrm{~V})$ and $\mathrm{CO}_{2}$ $(1.5 \mathrm{NL} / \mathrm{min})$. Before activation, disks were fixed on glass slides, and grafts were wrapped in grounded aluminum foil. Discs were treated for $7 \mathrm{~s}$ each. The lumen of grafts 


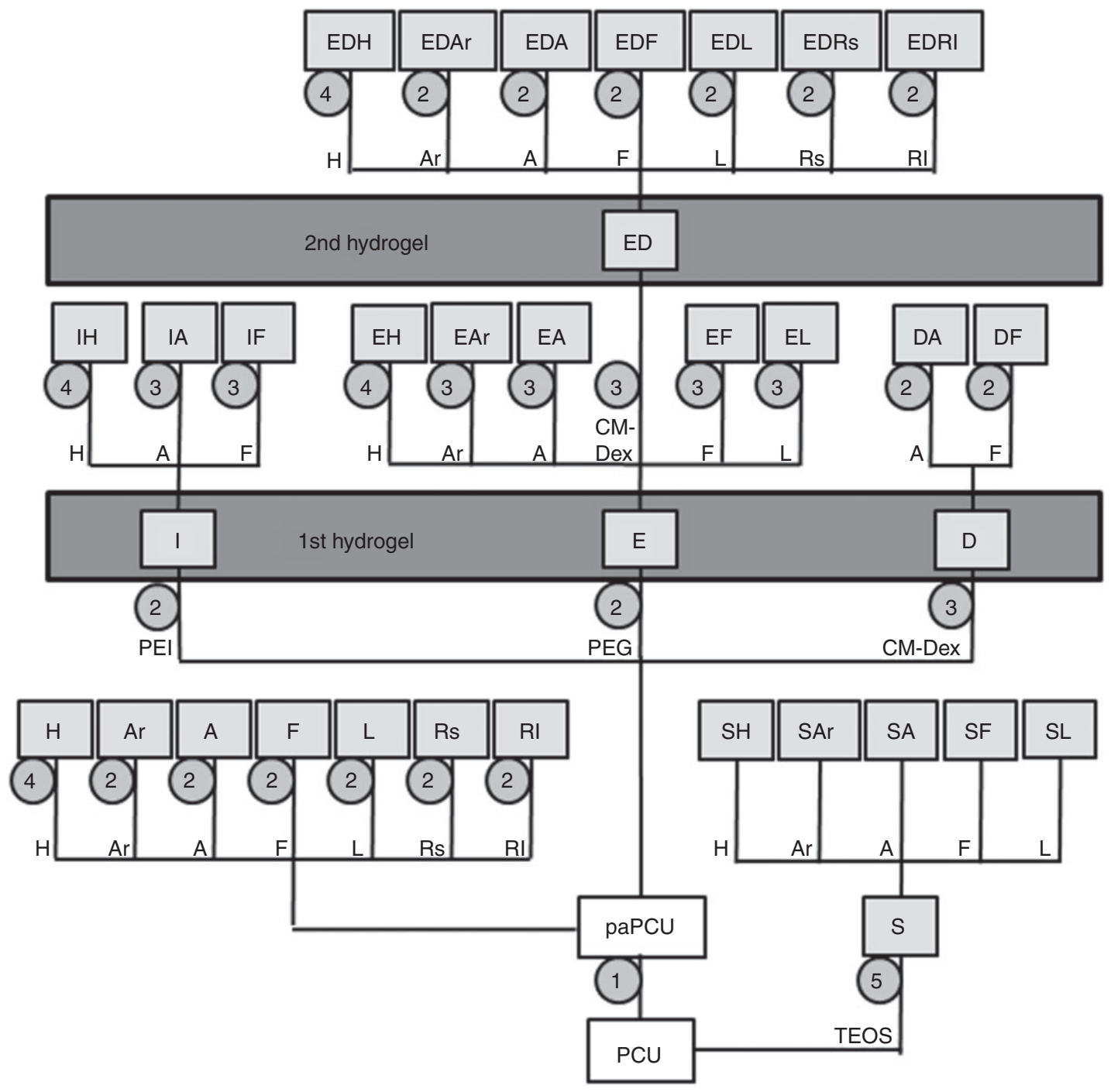

Figure I. Manufacturing of different chemical modifications of PCU.

PCU was modified using different procedures ( -5 , grey encircled) to introduce hydrogels (PEI, polyethyleneimine; PEG, poly(ethylene glycol) bis(amine)), cell-active molecules (Ar, argatroban; A, Antithrombin III; F, fibrinogen; L, laminin-nonapeptide; Rs, RI, short and long RGD-peptides; H, modified heparin), and carboxymethyl-dextran (CM-Dex). End products are framed at the end of the connecting lines. For details of the procedures and composition of the end products see "Materials and methods" section.

was treated with $\mathrm{N}_{2}$-plasma from one end and $\mathrm{CO}_{2}$ gas from the opposite site for $20 \mathrm{~s}$ once. The distance of the nozzle to the PCU was $0.5 \mathrm{~cm}$. After $45 \mathrm{~min}$ on air, plasma-activated PCU-samples (paPCU) were incubated with $0.9 \% \mathrm{NaCl}$ solution $\left(2 \mathrm{~h}, 50^{\circ} \mathrm{C}\right)$, washed in water and immediately modified.

Procedure 2. NHS/EDC chemistry (N-hydroxysuccinimide/1-ethyl-3-(3-dimethylaminopropyl) carbodiimide $)^{33}$ was used to activate modified PCU-samples (paPCU, CM-Dex immobilized surfaces). Samples were incubated with NHS/EDC $(0.1 \mathrm{M} / 0.1 \mathrm{M})$ in aqueous media (room temperature, RT, $20 \mathrm{~min}$ ), rinsed with water and processed.
Procedure 3. A ( $4 \mathrm{IU} / \mathrm{mL}), \mathrm{Ar}, \mathrm{L}$, and $\mathrm{F}$ (each, $0.4 \mathrm{mg} / \mathrm{mL}$ ) and CM-Dex $(4 \mathrm{mg} / \mathrm{mL})$ in c-buffer were incubated in NHS/EDC (0.1 M/0.2 M) (20 min, RT), and filled up to the 4-fold volume of c-buffer. Modified PCU samples were incubated in $\mathrm{A}$, Ar, L, and F solutions $\left(19 \mathrm{~h}, 4^{\circ} \mathrm{C}\right)$ or CM-Dex solution (2h, RT).

Procedure 4. Sodium cyanoborhydride $\left(\mathrm{NaBH}_{3} \mathrm{CN}\right)$ was used to immobilize modified heparin $(\boldsymbol{H})$ (see above). Modified heparin $(1 \mathrm{mg} / \mathrm{mL})$ was dissolved in $\mathrm{NaBH}_{3} \mathrm{CN}(3 \mathrm{mg} / \mathrm{mL})$ in $0.15 \mathrm{M} \mathrm{NaCl}$ and incubated with modified PCU-samples $\left(2 \mathrm{~h}, 50^{\circ} \mathrm{C}\right)$. After repeated short-time rinsing with water, the samples were dried and stored at $4{ }^{\circ} \mathrm{C}$ until biological testing. 
Procedure 5. Tetraethylorthosilicate (TEOS) was used as a precursor to polymerize on PCU-samples via sol-gel process. ${ }^{34}$ TEOS gas was generated by heating up to $80^{\circ} \mathrm{C}$ and fed in with Argon gas carrier $(10 \mathrm{NL} / \mathrm{min}$; $4500 \mathrm{~V})$ into the argon-plasma using the Plasmabrush ${ }^{\circledR}$ $(\boldsymbol{S})$. Disks were treated $(3 \times 7 \mathrm{~s})$. Immediately after polymerization of TEOS, disks were continuously moved in $10 \%$ 1,6-hexamethylenediisocyanate ${ }^{35}$ in diethylether $(72 \mathrm{~h}, \mathrm{RT})$ followed by diethylether washing $(5 \mathrm{~h})$ and repeated c-buffer washings. Thereafter, disks were incubated with A (1 IU / mL), modified heparin, Ar, $\mathrm{L}$, and $\mathrm{F}$ (each, $0.1 \mathrm{mg} / \mathrm{mL}$ ) (dissolved in c-buffer) over night at $4^{\circ} \mathrm{C}(\boldsymbol{S} \boldsymbol{A}, \boldsymbol{S H}, \boldsymbol{S A r}, \boldsymbol{S L}, \boldsymbol{S F})$.

Hydrogels of PEI $(\boldsymbol{I})$ and PEG $(\boldsymbol{E})$ were produced by crosslinking PEI $(3 \mathrm{mg} / \mathrm{mL}$ in c-buffer) $(2 \mathrm{~h}, \mathrm{RT})$ and PEG $(1 \mathrm{mg} / \mathrm{mL}$, in $0.6 \mathrm{M}$ potassium sulfate and cbuffer) $\left(2 \mathrm{~h}, 50^{\circ} \mathrm{C}\right)^{23}$ with activated PCU samples (procedure 2). For immobilization of CM-Dex, CM-Dex $(4 \mathrm{mg} / \mathrm{mL})$ was activated (procedure 3) and incubated with paPCU $(\boldsymbol{D})$ and $E$-modified PCU $(\boldsymbol{E D})(2 \mathrm{~h}, \mathrm{RT})$.

Covalent binding of cell-active molecules. The end product of chemical modification strategies consisted of covalent binding of argatroban (Ar), ATIII (A), fibrinogen (F), laminin-nonapeptide (L), short (Rs), and long (Rl) RGD-peptides. Molecules were either dissolved in c-buffer $(\mathrm{A}(1 \mathrm{IU} / \mathrm{mL}), \mathrm{Ar}, \mathrm{L}, \mathrm{Rs}, \mathrm{R} 1, \mathrm{~F}$ (each, $0.1 \mathrm{mg} / \mathrm{mL})$ ) and incubated with activated paPCU, activated $E D$-modified PCU, activated $D$-modified PCU (procedure 2) (19h, $\left.4^{\circ} \mathrm{C}\right)[\boldsymbol{A r}, \boldsymbol{A}, \boldsymbol{F}, \boldsymbol{L}, \boldsymbol{R s}, \boldsymbol{R l}, \boldsymbol{E D} \boldsymbol{A r}$, $E D A, E D F, E D L, E D R s, E D R l, D A, D F]$, or activated using procedure 3 and incubated $\left(19 \mathrm{~h}, 4^{\circ} \mathrm{C}\right)$ with $E$ - and $I$-modified PCU-samples $[\boldsymbol{E} \boldsymbol{A} \boldsymbol{r}, \boldsymbol{E} \boldsymbol{A}, \boldsymbol{E F}, \boldsymbol{E} \boldsymbol{L}, \boldsymbol{I} \boldsymbol{A}, \boldsymbol{I} \boldsymbol{F}]$. Heparin was immobilized onto paPCU, $S-, E-, E D-$, and $I$-modified PCU samples using procedure $4[\boldsymbol{H}$, $E H, E D H, I H]$.

Each end product (framed within Figure 1) was thoroughly rinsed with water, dried, and stored at $4^{\circ} \mathrm{C}$ until blinding and biological testing. Blinded test samples were washed (ethanol, 70\%; sterile PBS), and fixed into 96-well microplate $\left(\mathrm{Nunc}^{\circledR}\right.$, Wiesbaden, Germany) with sterile steel rings. Tissue-cultured polystyrene (TCP) (Nunc) was the reference for biological testing. ${ }^{36}$

\section{Cytocompatibility}

Human saphenous vein ECs (HSVEC) were cultured in growth medium with serum (GMS, Medium 199, 10\% fetal calf serum, L-Glutamine (PAA Laboratories, Pasching, Austria), Supplement Pack (PromoCell, Heidelberg, Germany)). ${ }^{36}$ Informed consent was obtained from cell donors. The protocol was approved by the local human ethics committee (no. 99/133). For adhesion (proliferation) tests, 66,000 (17,000) HSVECs/ $\mathrm{cm}^{2}$ were seeded onto non-coated test samples or fibronectin-coated $(10 \mu \mathrm{g} / \mathrm{mL}) \mathrm{TCP}$ for $24 \mathrm{~h}$ (3-7 days). Cells were fed with fresh GMS (days 3 and 5), harvested by addition of trypsin/EDTA (Promocell) on days 3, 5, and 7, and counted (CASY-TTC, Roche, Mannheim, Germany).

\section{Hemocompatibility}

Human citrated venous blood was drawn from male healthy volunteers with written consent as per institutional ethics guidelines (no. 10-101-0159), and centrifuged $\left(300 \mathrm{~g}, 15 \mathrm{~min}, 37^{\circ} \mathrm{C}\right)$. Platelet-rich plasma (PRP) was transferred into a polypropylene syringe containing $10 \%$ aqueous citrate dextrose, and centrifuged again. Platelets were counted (Neubauer haemocytometer), double-stained (monoclonal antiCD41-FITC/anti-CD62P-APC antibodies, BD Biosciences, Erembodegem, Belgium) $\left(24 \mathrm{~h}, 4^{\circ} \mathrm{C}\right)$ and analyzed using flow cytometry. Only PRP preparations with $<10 \% \mathrm{CD} 41+/ \mathrm{CD} 62 \mathrm{P}+$ platelets were used for platelet adhesion tests. PRPs $\left(5 \times 10^{7}\right.$ platelets $\left./ 0.3 \mathrm{~cm}^{2}\right)$ were coincubated with test samples $\left(30 \mathrm{~min}, 37^{\circ} \mathrm{C}\right)$. Non-adherent platelets were removed by careful washing with PBS. Adherent platelets were fixed (10\% paraformaldehyde, RT, $10 \mathrm{~min})$, permeabilized $(0.5 \%$ TritonX-100 in PBS, RT, 5 min), washed with PBS, stained with rhodamine-phalloidin (Molecular Probes, ThermoFisher, Darmstadt, Germany) (100 nM, RT, $30 \mathrm{~min}$ ), and visualized using fluorescence microscopy (Leica DMRBE, Biberach, Germany). Cell density was estimated using a platelet score $(1,<20 \% ; 2,20-50 \%$; $3,>50 \%$ surface coverage) (Figure 2(a) to (c)). Platelet score was evaluated in independent experiments using five different donors by three technicians in a blinded fashion. A mean platelet score $<1.5$ was defined as sufficient hemocompatible.

\section{Effect of shear stress}

Modifications with the best EC adhesion properties were used to analyze the effect of shear stress on cell adhesion in a bioreactor (DUALIS). Details for bioreactor processing see Riescher et al. ${ }^{37}$ HSVECs $\left(2-6 \times 10^{6}\right)$ were resuspended in $2 \mathrm{~mL}$ of GMS and added into the modified grafts. Fibronectin-coated grafts made of Elastollan ${ }^{\circledR} 1180 \mathrm{~A}$ (BASF, Ludwigshafen, Germany) were used as a positive control. ${ }^{37}$ The grafts were perfused with a pulsatile flow for $72 \mathrm{~h}$. Then, grafts were removed from the bioreactor, fixed with paraformaldehyde, and stained with 4',6-Diamidin-2'-phenylindol-dihydrochloride (DAPI). The grafts were separated into pieces of $1 \mathrm{~cm}$ of length, opened longitudinally and fixed on a glass slide. The nuclei of the adherent cells were visualized with the fluorescence microscope $(10 \times$ magnifications) and 

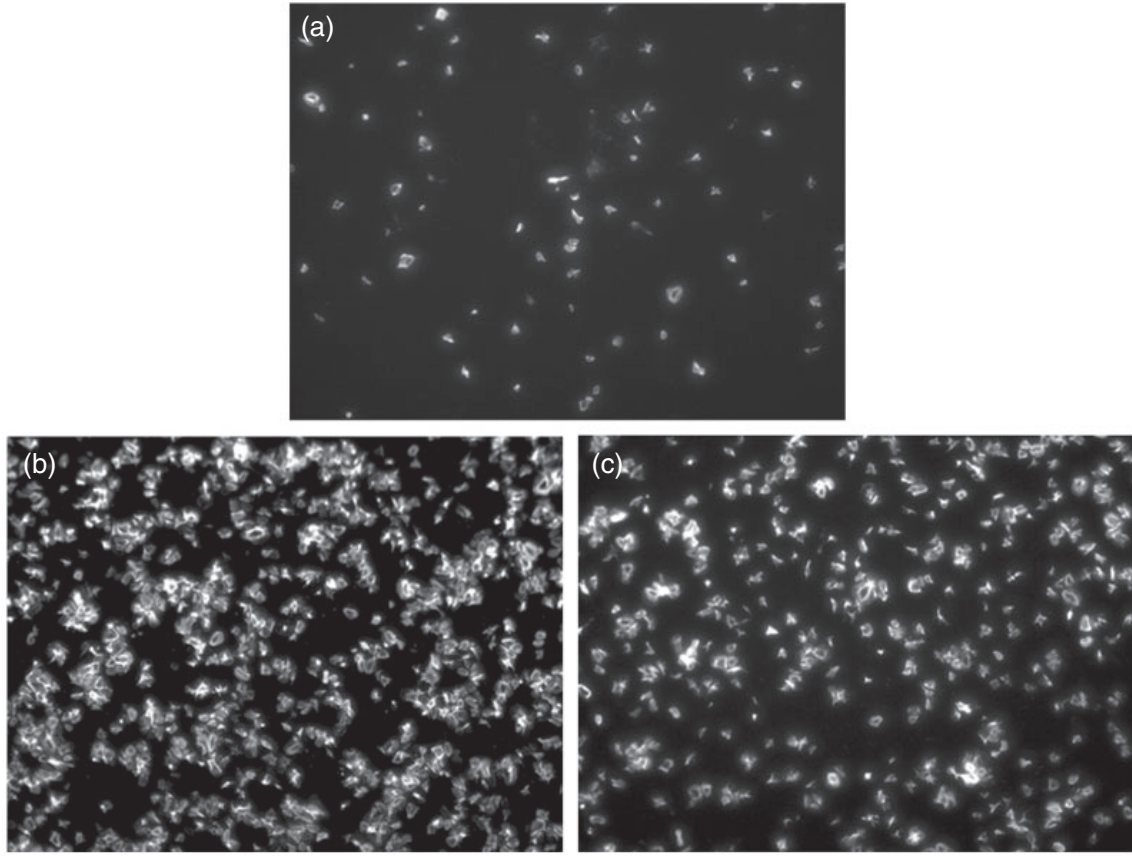

Figure 2. Estimation of the platelet score.

Different amounts of isolated platelets ((a) $6 \times 10^{6}$; (b) $2.5 \times 10^{7}$; (c) $5 \times 10^{7}$ per $0.3 \mathrm{~cm}^{2}$ ) were seeded onto tissue-cultured polystyrene (TCP) for $30 \mathrm{~min}$, fixed with formaldehyde, permeabilized, stained with rhodamine-phalloidin and visualized using fluorescence. Platelet score was defined as surface coverage of stained platelets: (a) platelet score I, <20\%; (b) platelet score 2, 20-50\%; (c) platelet score $3,>50 \%$ surface coverage.

quantified using ImageJ software. Other sections of the grafts were stained with rhodamine-phalloidin (see above) and with fluorescein isothiocyanate (FITC)conjugated monoclonal anti-human CD31 antibody/ Ancell, Bayport, MN). Furthermore, a third part of the grafts was used for scanning electron microscopy (SEM) as described by Lehle et al. ${ }^{38}$

\section{Statistics}

Data were presented as mean \pm standard deviation (SD). Due to non-normality of the data, the Wilcoxon-Signed-Rank-Test (Sigma-Stat, SPSS, Chicago, IL) was used after passing the FriedmanTest (Sigma-Stat, SPSS, Chicago, IL). $p$-Values $\leq 0.05$ were considered significant. All analyses in the static cell culture were done with six EC and five platelet donors. Other details see text.

\section{Results}

\section{Screening of different chemical modifications}

Platelet adhesion (Figure 3(a)) was high for TCP and untreated PCU with a platelet score of $2.9 \pm 0.3$ and $2.2 \pm 0.5$, respectively. After chemical modification of PCU, 15 different strategies $(43 \%)$ presented a significant reduction of the mean platelet score $(1.3 \pm 0.6$, $p \leq 0.001$ vs. untreated PCU) (white bars, Figure 3(a)). As shown in Figure 3(b), TCP allowed high EC coverage $(100 \%)$, while the cell density was significantly reduced for untreated PCU ( $48 \pm 13 \%$ of TCP, $p \leq 0.001)$. Cell adhesion onto PCU was significantly improved in 19 modifications (54\%) (white bars, Figure 3(b)) $(p \leq 0.05$ vs. untreated PCU). Nine modifications $(26 \%)$ unified both anti-thrombogenic and EC-adhesive characteristics (shaded boxes, Figure 3(a) and (b)).

As shown in Figure 3(a) and (b), plasma activation of PCU (paPCU) did not reduce platelet adhesion (platelet score $>2, p>0.05$ ) but significantly increased $\mathrm{EC}$ adhesion $(p=0.024)$. However, no additional treatment enabled a further increase in EC density compared to paPCU. Only the direct coupling of heparin (H) improved EC coverage; however, without statistical significance $(p=0.063$, compared to paPCU). Surprisingly, binding of fibronectin to PEI hydrogels even reduced EC density (IF). Polymerization of TEOS and further modifications ( $\mathrm{S}, \mathrm{SH}, \mathrm{SAr}, \mathrm{SA}, \mathrm{SF}$, SL) did not affect EC-adhesive properties, but instead increased platelet score $(>2)$. Therefore, this procedure was not pursued further. Further contemplation focused on anti-thrombogenic surfaces (platelet score $<1.5$, Figure 3(a)). PEG-based hydrogel coatings (E, ED) significantly reduced thrombogenicity (E, $p=0.009$; ED, $p=0.029$; compared to untreated PCU). Coupling of PEI (I) and CM-Dex (D) did not 

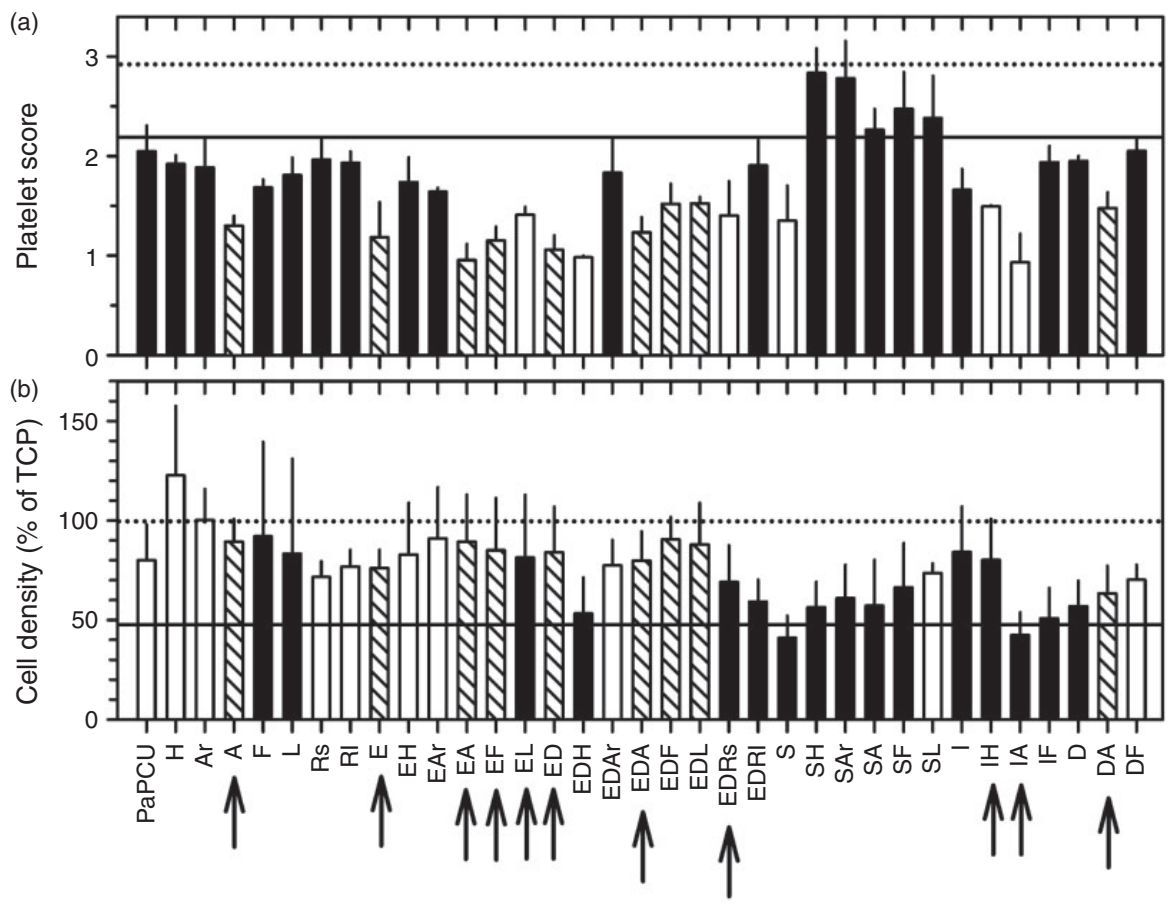

Figure 3. Biological screening of different chemical modifications.

Different chemical modifications (for abbreviations and manufacturing protocol see "Materials and methods" section) were tested for their (a) hemocompatibility and (b) cytocompatiblilty. Platelet adhesion was estimated via platelet score (5 donors). Endothelial cells (8 donors) were cultivated for $24 \mathrm{~h}$ and counted with CASY-TTC. Cell counts were specified in $\%$ of TCP (tissue-cultured polystyrene). Solid black line, untreated PCU; dotted black line, TCP; filled boxes, no significant alteration compared to untreated PCU; white boxes, significant reduction of platelet score $(<1.5)$ or increase of EC density compared to untreated PCU; shaded boxes, concerning modifications combined both anti-thrombogenicity and endothelial cell adhesion; arrows, these modifications were used to demonstrate reproducibility of the manufacturing process. Data are presented as mean and standard deviation.

improve anti-thrombogenicity (platelet score $>1.5$ ). Covalent binding of ATIII-A, EA, EDA, IA, DA-improved anti-thrombotic properties of untreated PCU (platelet score $<1.5$ ) (each, $p \leq 0.001$ compared to untreated PCU). Comparing preconditioning treatment with respective ATIII modifications showed a significant reduction for $\mathrm{A}$, IA, and DA (paPCU vs. A, $p=0.004$; I vs. IA, $p=0.010$; D vs. DA, $p=0.002$ ). A benefit in anticoagulant function of Argatroban (Ar, EAr, EDAr) and modified heparin $(\mathrm{H}, \mathrm{EH}, \mathrm{EDH}, \mathrm{IH})$ failed. Additional binding of EC-adhesive proteins/peptides such as fibronectin did not affect the anti-thrombotic properties of respective pretreated PCUs (F, EF, EDF, IF, DF).

\section{Reproduction of the manufacturing process}

Eleven of thirty-five modifications were tested for reproducibility of the manufacturing process. Selection criteria included (a) anti-thrombogenicity (Figure 3(a)), (b) easy manufacturing, (c) preservation of EC-adhesiveness (Figure 3(b)). Manufacturing process was repeated three times in an independent fashion. As shown in Table 1, platelet adhesion was highly variable for different preparations of A, E, EF, ED, EH, and DA. Reproduction of low platelet scores was documented for EA, EL, EDA, EDRs, and IA (foregrounded in grey, Table 1). As shown in above, EC adhesiveness was only adequate for $7 / 11$ selected modifications. EL, EDRs and IH showed high variability in EC coverage. IA prevented EC adhesion. These characteristics were reproducible for $\mathrm{A}, \mathrm{E}, \mathrm{IH}$ (maintenance of EC adhesiveness), and IA (prevention of EC adhesion) (foregrounded in grey, Table 1).

\section{EC proliferation}

Improved cytocompatibility of biomaterials was also defined by an increase in the proliferative activity of EC (Figure 4). Highest cell density was detected for TCP $(n=8, p \leq 0.001)$. Instead, cell density was by a factor of 2 significantly lower on untreated PCU $(p=0.001)$. Additional modifications either completely suppressed proliferation (EA, ED, EDA, EDRs, IA), or permitted an increase in cell count over 5 days but without a synergistic effect compared to untreated PCU (A, E, EF, EL, IH, DA). 
Table I. Reproduction of the manufacturing process of II modifications.

\begin{tabular}{|c|c|c|c|c|c|c|c|c|}
\hline \multirow[b]{2}{*}{ Preparation } & \multicolumn{4}{|c|}{ EC adhesion ( $\%$ of TCP) } & \multicolumn{4}{|c|}{ Platelet adhesion (platelet score) } \\
\hline & $I^{\text {st }}$ & $2^{\text {nd }}$ & $3^{\text {rd }}$ & $4^{\text {th }}$ & $I^{\text {st }}$ & $2^{\text {nd }}$ & $3^{\text {rd }}$ & $4^{\text {th }}$ \\
\hline \multicolumn{9}{|l|}{ Modification } \\
\hline Untreated & $50 \pm 10$ & $48 \pm 13$ & $60 \pm 19$ & $61 \pm 20$ & $2.5 \pm 0.5$ & $2.2 \pm 0.3$ & $2.7 \pm 0.1$ & $2.2 \pm 0.2$ \\
\hline A & $89 \pm 11$ & $55 \pm 23$ & $66 \pm 23$ & $67 \pm 13$ & $\mathrm{I} .3 \pm 0.6$ & $\mathrm{I} .7 \pm 0.5$ & $\mathrm{I} .8 \pm 0.7$ & $\mathrm{I} .8 \pm 0.8$ \\
\hline $\mathrm{E}$ & $76 \pm 9$ & $56 \pm 16$ & $75 \pm 18$ & $56 \pm 18$ & $\mathrm{I} .2 \pm 0.5^{*}$ & $1.2 \pm 0.6^{*}$ & $2.3 \pm 0.8$ & $1.2 \pm 0.5^{*}$ \\
\hline EA & $89 \pm 24$ & $29 \pm 10$ & $65 \pm 9$ & $53 \pm 43$ & $0.9 \pm 0.6$ & $\mathrm{I} . \mathrm{I} \pm 0.6$ & $1.6 \pm 0.7$ & $1.2 \pm 0.5$ \\
\hline EF & $85 \pm 26$ & $46 \pm 13$ & $80 \pm 15$ & $47 \pm 21$ & $\mathrm{I} .2 \pm 0.6$ & $\mathrm{I} . \mathrm{I} \pm 0.6$ & $1.9 \pm 1.0$ & $\mathrm{I} . \mathrm{I} \pm 0.7$ \\
\hline EL & $81 \pm 31$ & $49 \pm 18$ & $73 \pm 11$ & $56 \pm 13$ & $\mathrm{I} .4 \pm 0.5$ & $1.2 \pm 0.6$ & $\mathrm{I} . \mathrm{I} \pm 0.6$ & $\mathrm{I} .2 \pm 0.7$ \\
\hline ED & $84 \pm 23^{8}$ & $39 \pm 19$ & $76 \pm 22^{8}$ & $27 \pm 17$ & $\mathrm{I} . \mathrm{I} \pm 0.5^{*}$ & $\mathrm{I} . \mathrm{I} \pm 0.6^{*}$ & $1.8 \pm 0.7$ & $1.0 \pm 0.7^{*}$ \\
\hline EDA & $80 \pm 15^{8}$ & $55 \pm 21^{\&}$ & $54 \pm 12^{\&}$ & $24 \pm 10$ & $1.2 \pm 0.7$ & $0.8 \pm 0.3^{\#}$ & $1.2 \pm 0.4$ & $1.0 \pm 0.6$ \\
\hline EDRs & $69 \pm 18$ & $14 \pm 4^{* \& \#}$ & $74 \pm 14$ & $31 \pm 5^{* \#}$ & $\mathrm{I} .5 \pm 0.7$ & $0.6 \pm 0.5^{\#, *}$ & $\mathrm{I} .5 \pm \mathrm{I} . \mathrm{I}$ & $1.0 \pm 0.5$ \\
\hline $\mathrm{IH}$ & $80 \pm 21$ & $74 \pm 18$ & $70 \pm 14$ & $70 \pm 15$ & $\mathrm{I} .5 \pm 0.7$ & $1.2 \pm 0.5^{*}$ & $1.8 \pm 0.8$ & $\mathrm{I} . \mathrm{I} \pm 0.6^{*}$ \\
\hline IA & $43 \pm 11$ & $31 \pm 13$ & $19 \pm 10$ & $27 \pm 18$ & $0.9 \pm 0.5^{8}$ & $0.9 \pm 0.5^{\&}$ & $0.9 \pm 0.5^{8}$ & $1.7 \pm 0.6$ \\
\hline DA & $63 \pm 14$ & $27 \pm 27^{* \#}$ & $75 \pm 21$ & $42 \pm 24$ & $\mathrm{I} .4 \pm \mathrm{I} .0$ & $1.6 \pm 1.0$ & $2.0 \pm 1.0$ & $\mathrm{I} .5 \pm \mathrm{I} .3$ \\
\hline
\end{tabular}

Data are mean \pm standard deviation. Abbreviations of the modifications are described in the "Materials and methods" section and Figure I. Each preparation $\left(I^{\text {st }}\right.$ to $\left.4^{\text {th }}\right)$ was done independently in a triple approach.

EC: endothelial cell; TCP: tissue culture polystyrole (reference material).

Statistics: *significant vs. $3^{\text {rd }}$ preparation; ${ }^{\#}$ significant vs. $1^{\text {st }}$ preparation; ${ }^{\&}$ significant vs. $4^{\text {th }}$ preparation.

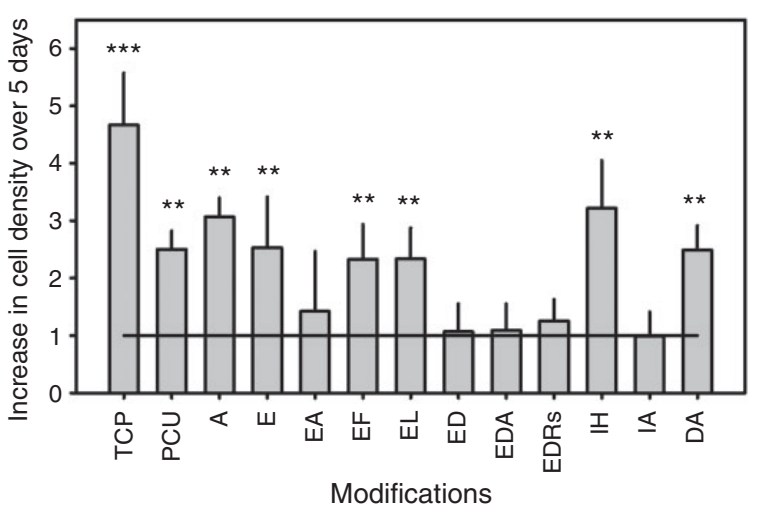

Figure 4. Proliferation of endothelial cells on the surface of selected modifications.

Endothelial cells $\left(17,000\right.$ per $\left.\mathrm{cm}^{2}\right)$ were seeded onto tissue-cultured polystyrene (TCP), untreated PCU and I I/35 modified test samples (for abbreviations and manufacturing protocol see "Materials and methods" section) for 5 days. Cells were counted and displayed relative to the cell count on the day of seeding (solid line). ${ }^{* * *} p \leq 0.001 ; * * p \leq 0.01$ compared to the cell count on the day of seeding. $\# p=0.00 \mathrm{I}$ comparing cell counts on day 5 after seeding on TCP and untreated PCU. Data are presented as mean and standard deviation from eight cultures.

\section{Effect of shear stress}

Modifications A, E, and IH combined benefits such as adequate $\mathrm{EC}$ adhesion and proliferation, and satisfactory reproducibility. These properties might enable cell seeding of vascular synthetic grafts in a bioreactor under pulsatile flow. ${ }^{29}$ After 3 days under shear stress (maximum flow, 600-800 mL/min; laminar flow; shear force, $6 \mathrm{dyn} / \mathrm{cm}^{2}$ ), cell density on fibronectin-coated Elastollan ${ }^{\circledR}$ grafts (positive control) was significantly higher than on untreated PCU grafts (Figure 5(a)). Nuclei from adherent cells on Elastollan ${ }^{\circledR}$ and untreated PCU showed an oval shape. The circularity (relative unit 1 represents a circle) of the nuclei described its roundness (Figure 5(b)). Adherent EC on the luminal surface of the Elastollan ${ }^{\circledR}$ graft formed a complete cell monolayer. The cells arranged in flow direction (Figure 5(c)). In contrast, pulsatile flow removed the integrity of adherent EC on untreated PCU grafts. As a result, cells detached and aggregated. Only small colonies remained adherent on the polymer surface.

In six independent experimental set-ups, modified PCU grafts (A, E, IH) were endothelialized and exposed to shear stress for 3 days. Immediately after induction of pulsatile flow EC completely detached from the surface of E-modified grafts. After 3 days, no EC was detected on these grafts. In contrast, EC remained attached on the surface of the other modified grafts $(\mathrm{A}, \mathrm{IH})$. As shown in Figure 6, EC formed a monolayer with its typical "cobblestone morphology". However, the cells differed in their size. Modification IH presented more cells with larger volume (cell swelling) (Figure 6(a)). EC on A-modified grafts were irregularly arranged (Figure 6(b)). In any case, cell arrangement in flow direction failed. In addition, the integrity of the monolayer was disrupted as shown in 
(a)

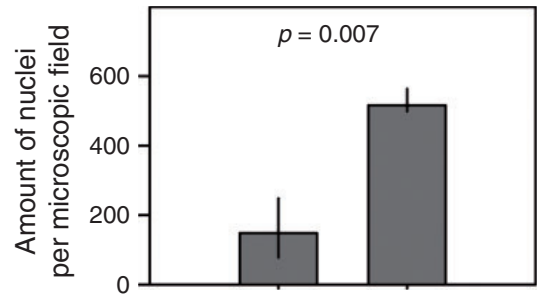

(b)
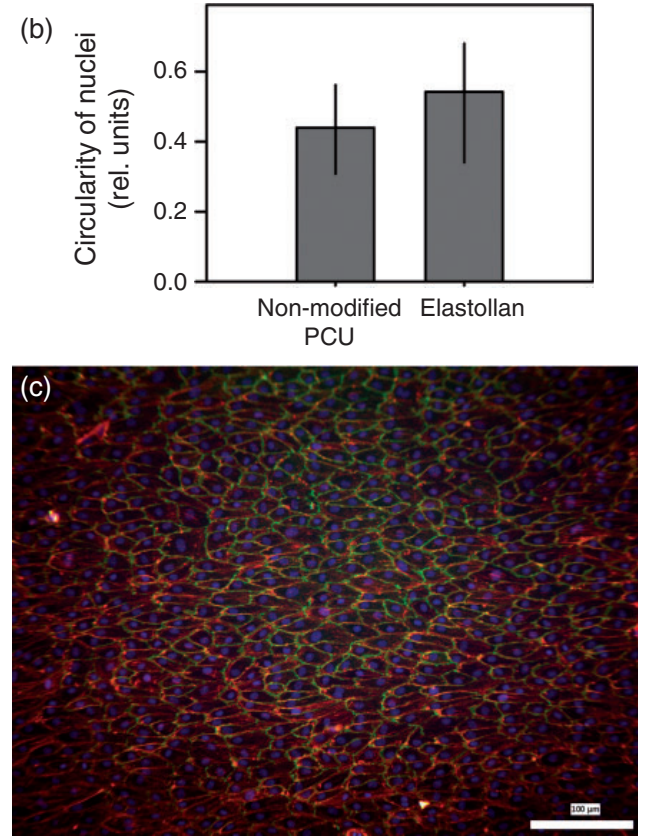

Figure 5. Effect of shear stress on the cell adhesion into grafts made of Elastollan ${ }^{\circledR}$ and untreated PCU. Details for usage of the bioreactor see reference \#29 and text. After 3 days under pulsatile flow, cell density on fibronectin-coated Elastollan ${ }^{\circledR}$ grafts (positive control) was significantly higher than on untreated PCU grafts (a). Nuclei from adherent cells were round (circularity; I represents a circle) (b). Endothelial cells formed a confluent monolayer. Cells arranged in flow direction (c).

Immunofluorescent staining of fixed cells: blue, DAPI, red, rhodamine-phalloidin; green CD3I-FITC.

representative REM photographs in Figure 6(c). The median cell density was quantified by computation of DAPI-stained nuclei per microscopic field (Figure 6(d)). Median cell density on modifications A and $\mathrm{IH}$ was about half of the positive control (high variation of cell counts). In detail, only one of six EC cultures showed good cell attachment on A-grafts, and three of six EC cultures formed a monolayer on IHgrafts. There was no difference in the circularity of the nuclei from cells on Elastollan ${ }^{\circledR}$, A-graft, and IH-graft. The shape of the nuclei of remaining cells on E-grafts was deformed - a sign of absent viability (Figure 6(e)).

\section{Discussion}

In this study, a multitude of chemical modifications was produced to reduce thrombogenicity and to improve cytocompatibility of PCU. Three of 35 modifications (A, E, IH) fulfilled biological improvements including reduced platelet adhesion, stable EC adhesion and proliferation, and a reproducible manufacturing process. However, under shear stress, the benefit of good EC adhesion/proliferation and satisfactory reproducibility disappeared.

Modification of the PCU surface chemistry combining reduced platelet adhesion/activation and increasing endothelial attachment whilst preserving the mechanical properties would be an elusive goal. Here, 9/35 modifications met both biological requirements. A promising approach was the usage of PEG-based hydrogel coatings alone or conjugated with ATIII and ECM proteins/peptides. PEG is biocompatible with blood and tissue, nontoxic to cellular systems, nonimmunogenic, an excellent conjugate for polymer graft materials, and has been approved by the FDA. ${ }^{39,40}$ Therefore, PEG hydrogels have been widely used as a supporting matrix in almost every field of tissue engineering (nerve, cartilage, liver, pancreas, bladder, skin, heart). The reduced platelet binding capacity resulted from its inert surface and low protein adsorption. ${ }^{41,42}$ The additional binding of anticoagulants had no synergistic anti-thrombogenic effect. Furthermore, the PEGbased hydrogels allowed EC adhesion. Single coating with PEG-diamine hydrogels even allowed EC proliferation. Furthermore, the manufacturing process of PEG-diamine hydrogel coating to plasma-activated PCU was highly reproducible. Similar results were presented for smooth muscle cell attachment to the surface of the PEG-genipin hydrogels ${ }^{17}$ and cultivation of neural cells. ${ }^{4-45}$ Additional conjugation with ECM peptides/proteins (e.g. RGD-peptides, fibronectin, laminin) had no synergistic effect on EC adhesion. This was in contrast to other studies using functionalized PEG hydrogels. Binding of RGD increased migration/adhesion of smooth muscle cells and allowed the construction of artificial blood vessels. ${ }^{46,47} \mathrm{We}$ supposed that the heterogeneous data from functionalized PEGhydrogels resulted from different preparation strategies of PEG-based hydrogels, various conjugation protocols and the pretreatment and type of the underlying polymer. Nevertheless, the potential of EC adhesion might further aggravate bacterial contamination to reduce risk of infection of such blood contacting surfaces. ${ }^{48}$

Of special interest for improved antithrombogenicity of cardiovascular devices was the immobilization of anticoagulants (e.g. heparin). In contrast to other studies, our binding strategies of heparin and Argatroban suppressed their anticoagulant function. Independent of pretreatment process (except silanization), conjugation with ATIII resulted in a reduced platelet adhesion. The manufacturing process that ensures active ATIII function was highly reproducible for PEG-based and 

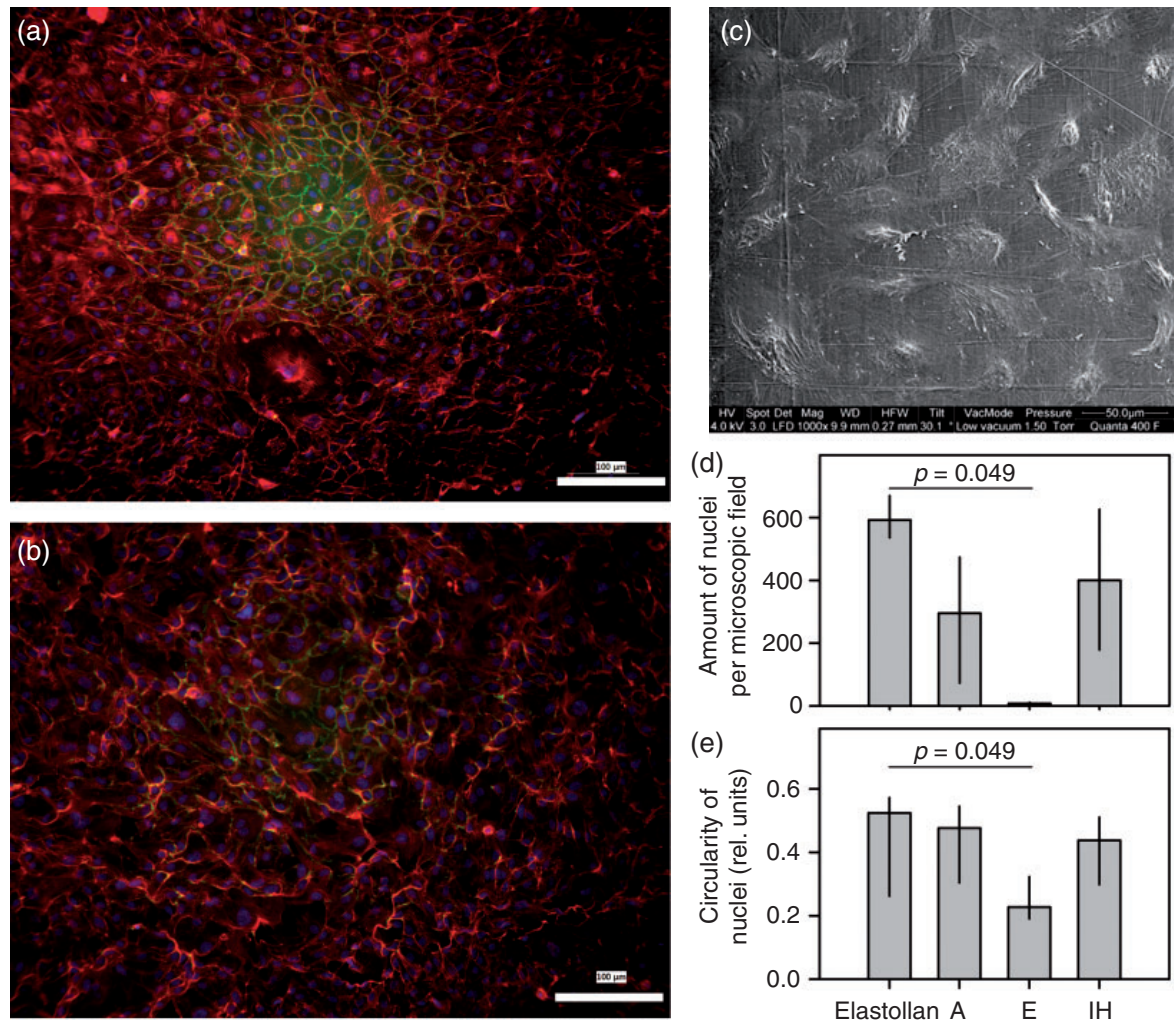

Figure 6. Effect of shear stress on endothelial cell coverage on modified PCU.

For details of protocol and abbreviations see "Materials and methods" section. After 3 days, endothelial cells formed a cobblestonelike monolayer on the luminal surface of $\mathrm{IH}$ - (a) and A-grafts (b). However, arrangement of the cells was different to Elastollan ${ }^{\circledR}$ grafts (Figure 4(c)). Staining was described in legend of Figure 4. Integrity of the monolayer was disrupted as shown in a representative SEM photograph from IH-grafts (c). Cell density (verified as described in legend of Figure 5) (d) and circularity of the nuclei (e) differed to data from Elastollan ${ }^{\circledR}$ grafts.

PEI hydrogels. The primary goal of this study was the creation of surfaces that prevent platelet adhesion and allow EC adherence as well. Therefore, EC-adhesive properties of $4 / 5$ ATIII binding strategies were analyzed. Unfortunately, ATIII binding strategy to PEG, PEG-CM-Dex, and CM-Dex hydrogels resulted in high variability of EC coverage. Furthermore, the PEI hydrogels conjugated with ATIII showed cytotoxic effects for EC. This was surprising because in this study PEI alone was not cytotoxic. Nevertheless, under in vitro conditions PEIs induced molecular weight dependent cytotoxic effects. ${ }^{49}$ There was no convincing explanation for cytotoxic effects of ATIIImodified PEIs. Finally, only direct coating of ATIII to plasma-activated PCU was a reproducible method for EC adhesion, but critical for reproducible low platelet adhesion (as discussed above).

The last interesting surface modification in this context comprised PEI hydrogel in combination with heparin binding. EC not only adhered and proliferated on PEI-heparin surfaces but also the manufacturing process was highly reproducible regarding EC adhesion.
Reproducibility of low platelet adhesion was acceptable. PEI-heparin nanogels were already used as gene delivery system in ovarian cancer cells ${ }^{50}$ that availed its excellent blood compatibility and low cytotoxicity. ${ }^{51}$

Only three PCU modifications (A, E, IH) fulfilled biological improvements including reduced platelet adhesion, stable EC adhesion and proliferation, and a reproducible manufacturing process. In cardiovascular tissue engineering applications, biocompatibility control also required analysis of shear forces on the retention of the cells on the luminal surface of the bioengineered vessel. Placement of endothelialized grafts into the arterial tree resulted in the removal of seeded cells because of high fluid shear forces applied to the graft lumen. Retention of a complete and stable EC layer on the lumen of vascular grafts in the presence of shear stress is an important prerequisite for longterm patency and prevention of thrombus formation. ${ }^{52}$ Therefore, a bioreactor was used to evaluate the strength of EC adhesion onto modified PCU surfaces within a vascular graft under pulsatile flow. In a previous study, it was already shown that a stable 
endothelialization can be achieved at pulsatile flow within the fibronectin-coated Elastollan ${ }^{\circledR}$ graft. ${ }^{37}$ As shown now, EC seeded onto this surface (reference material) not only formed a confluent monolayer but also rearranged to align themselves to the direction of flow, thus establishing a characteristic polarization that is observable both in vivo and in vitro. ${ }^{53-55}$ However, the Elastollan ${ }^{\circledR}$ material did not fulfill the mechanical properties required for usage in arterial grafts or a pump chambers in ventricular assist devices (data not shown). Instead, the PCU from the present study was more compatible as graft material, but after seeding of EC onto untreated and modified PCU grafts, shear stress caused loss of cell-cell-interactions of ECs which resulted in partial or complete removal of the cells. Similar results were shown by Inoguchi et al. ${ }^{30}$ Human umbilical vein ECs seeded onto compliant small-diameter grafts made of poly(L-lactide-co-epsilon-caprolactone) fiber meshes got lost after exposure to high shear forces $\left(>9 \mathrm{dyne} / \mathrm{cm}^{2}\right)$. The shear forces used in the present study $\left(6 \mathrm{dyn} / \mathrm{cm}^{2}\right)$ did not cause cellular damage because the EC monolayer on reference material remained stable and exhibited polygonal cobblestone morphology with aligned cells in flow direction. Therefore, it could be speculated, that the anchorage of the EC onto the selected modified PCU was too weak and the cells got lost under shear stress. Only PCU grafts with the IH-modification remained attached after 3 days under pulsatile flow. Nevertheless, the monolayer was incomplete and the cells were not arranged in flow direction. The denuded areas may lead to thrombus formation when implanted in the vascular network. ${ }^{52,56}$ Restricted polarization seems to be an indicator for missing shear force resistance. ${ }^{55}$ Attempts to seed ECs on current vascular prosthesis materials remains problematic. Despite different strategies to modify scaffold materials such as binding of cell-adhesive proteins such as fibronectin, laminin, and collagen VIII, the exposure to shear stress in vivo resulted in detachment of EC and clot formation. ${ }^{57,58}$ Promising results included titanium-coating ${ }^{37}$ and usage of nanocomposite biomaterial-based vascular grafts. ${ }^{59}$ Used PCU grafts were modified with RGD-peptides and demonstrated under static and dynamic conditions a relatively rapid endothelialization from endothelial progenitor cells. In addition to a compatible surface treatment for stable endothelialization, control of shear forces is inalienable in biocompatibility analysis of bioengineered grafts and vascular assist devices. ${ }^{55}$ Future studies must include not only static tests but also standardized bioreactor systems that produced physiological flow streams before implantation into animals or humans. ${ }^{60}$
The present study has some limitations. We used primary human endothelial cells instead of bloodderived progenitor cells ${ }^{59}$ that might be an attractive cell type to demonstrate in vivo endothelialization of implantable cardiovascular grafts. ${ }^{61}$ Furthermore, our study focused on the biological characteristics of the surface modifications. Due to the fact, that none of the introduced modifications fulfilled our requirements, physicochemical properties were not analyzed.

\section{Conclusion}

Bio- and hemocompatibility testing of plasma-treated and chemically modified biomedical-grade PCU exhibited promising surface modification that permitted platelet adhesion and improved endothelialization. However, improvement of EC adhesion as detected under static culture conditions failed after introduction of shear stress. This study suggests the importance of including mechanical forces in terms of endothelial retention and ability to resist circulating blood elements under physiologic conditions. Functionalization of PCU to promote stable endothelialization for bioengineered vascular grafts or ventricular pump chambers required further scientific input with special consideration of shear stress.

\section{Acknowledgements}

The authors gratefully acknowledge the excellent technical assistance of Sara Bergmann and Katrin Bielenberg.

\section{Declaration of Conflicting Interests}

The author(s) declared no potential conflicts of interest with respect to the research, authorship, and/or publication of this article.

\section{Funding}

The author(s) disclosed receipt of the following financial support for the research, authorship, and/or publication of this article: The study was supported by EFRE, European funds for regional development, Bavarian, Germany.

\section{References}

1. Guo JT, Ye YQ, Feng YK, et al. Studies on NO releasing from PCU grafted with endogenous NO donors. Polym Adv Technol 2010; 21: 759-766.

2. Xue L and Greisler HP. Biomaterials in the development and future of vascular grafts. J Vasc Surg 2003; 37: 472-480.

3. Daebritz SH, Fausten B, Hermanns B, et al. New flexible polymeric heart valve prostheses for the mitral and aortic positions. Heart Surg Forum 2004; 7: E525-532.

4. Szelest-Lewandowska A, Masiulanis B, Szymonowicz M, et al. Modified polycarbonate urethane: Synthesis, properties and biological investigation in vitro. J Biomed Mater Res A 2007; 82: 509-520. 
5. Chen KY, Kuo JF and Chen CY. Synthesis, characterization and platelet adhesion studies of novel ion-containing aliphatic polyurethanes. Biomaterials 2000; 21: 161-171.

6. D'Arrigo P, Giordano C, Macchi P, et al. Synthesis, platelet adhesion and cytotoxicity studies of new glycerophosphoryl-containing polyurethanes. Int $J$ Artif Organs 2007; 30: 133-143.

7. Gunatillake PA, Martin DJ, Meijs GF, et al. designing biostable polyurethane elastomers for biomedical implants. Austral J Chem 2003; 56: 545-557.

8. De S, Sharma R, Trigwell S, et al. Plasma treatment of polyurethane coating for improving endothelial cell growth and adhesion. J Biomater Sci Polym Ed 2005; 16: 973-989.

9. Wilson DJ, Rhodes NP and Williams RL. Surface modification of a segmented polyetherurethane using a lowpowered gas plasma and its influence on the activation of the coagulation system. Biomaterials 2003; 24: 5069-5081.

10. Pringle SD, Joss VS and Jones C. Ammonia plasma treatment of PTFE under known plasma conditions. Surf Interf Anal 1996; 24: 821-827.

11. Rhodes NP, Wilson DJ and Williams RL. The effect of gas plasma modification on platelet and contact phase activation processes. Biomaterials 2007; 28: 4561-4570.

12. Luna SM, Silva SS, Gomes ME, et al. Cell adhesion and proliferation onto chitosan-based membranes treated by plasma surface modification. J Biomater Appl 2011; 26: 101-116.

13. Wang $\mathrm{H}, \mathrm{Xu} \mathrm{M}, \mathrm{Wu} \mathrm{Z}$, et al. Biodegradable poly(butylene succinate) modified by gas plasmas and their in vitro functions as bone implants. ACS Appl Mater Interfaces 2012; 4: 4380-4386.

14. Zhang X, Xu B, Puperi DS, et al. Application of hydrogels in heart valve tissue engineering. J Long Term Eff Med Implants 2015; 25: 105-134.

15. Tibbitt MW and Anseth KS. Hydrogels as extracellular matrix mimics for $3 \mathrm{D}$ cell culture. Biotechnol Bioeng 2009; 103: 655-663.

16. Zhu JM. Bioactive modification of poly(ethylene glycol) hydrogels for tissue engineering. Biomaterials 2010; 31: 4639-4656.

17. Moffat KL and Marra KG. Biodegradable poly(ethylene glycol) hydrogels crosslinked with genipin for tissue engineering applications. J Biomed Mater Res B Appl Biomater 2004; 71: 181-187.

18. Pippig F and Holländer A. Hydrogel nanofilms for biomedical applications: Synthesis via polycondensation reactions. Macromol Biosci 2010; 10: 1093-1105.

19. Shkilnyy A, Gräf R, Hiebl B, et al. Unprecedented, low cytotoxicity of spongelike calcium phosphate/poly(ethylene imine) hydrogel composites. Macromol Biosci 2009; 9: 179-186.

20. Carrigan SD, Scott G and Tabrizian M. Rapid threedimensional biointerfaces for real-time immunoassay using hIL-18BPa as a model antigen. Biomaterials 2005; 26: 7514-7523.

21. Zhang R, Tang M, Bowyer A, et al. A novel $\mathrm{pH}$ - and ionic-strength-sensitive carboxy methyl dextran hydrogel. Biomaterials 2005; 26: 4677-4683.
22. Solouk A, Cousins BG, Mirahmadi F, et al. Biomimetic modified clinical-grade POSS-PCU nanocomposite polymer for bypass graft applications: A preliminary assessment of endothelial cell adhesion and haemocompatibility. Mater Sci Eng C Mater Biol Appl 2015; 46: 400-408.

23. Heller M, Kämmerer PW, Al-Nawas B, et al. The effect of extracellular matrix proteins on the cellular response of HUVECS and HOBS after covalent immobilization onto titanium. J Biomed Mater Res A 2015; 103: 2035-2044.

24. Kakinoki S and Yamaoka T. Single-step immobilization of cell adhesive peptides on a variety of biomaterial substrates via tyrosine oxidation with copper catalyst and hydrogen peroxide. Bioconjug Chem 2015; 26: 639-644.

25. Kuehn C, Dubiel EA, Sabra G, et al. Culturing INS-1 cells on CDPGYIGSR-, RGD- and fibronectin surfaces improves insulin secretion and cell proliferation. Acta Biomater 2012; 8: 619-626.

26. Nelson J, Scott WN, Allen WE, et al. Murine epidermal growth factor peptide (33-42) binds to a YIGSR-specific laminin receptor on both tumor and endothelial cells. J Biol Chem 1996; 271: 26179-26186.

27. Murugesan S, Xie $\mathbf{J}$ and Linhardt RJ. Immobilization of heparin: Approaches and applications. Curr Top Med Chem 2008; 8: 80-100.

28. Tan D, Zhang X, Li J, et al. Modification of poly(ether urethane) with fluorinated phosphorylcholine polyurethane for improvement of the blood compatibility. $J$ Biomed Mater Res A 2012; 100: 380-387.

29. Major TC, Brisbois EJ, Jones AM, et al. The effect of a polyurethane coating incorporating both a thrombin inhibitor and nitric oxide on hemocompatibility in extracorporeal circulation. Biomaterials 2014; 35: 7271-7285.

30. Inoguchi $H$, Tanaka $T$, Maehara $Y$, et al. The effect of gradually graded shear stress on the morphological integrity of a HUVEC-seeded compliant small-diameter vascular graft. Biomaterials 2007; 28: 486-495.

31. McArthur SL, McLean K, Kingshott P, et al. Effect of polysaccharide structure on protein adsorption. Colloids Surf B Biointerf 2000; 17: 37-48.

32. Klee D, Ademovic Z, Bosserhoff A, et al. Surface modification of poly(vinylidenefluoride) to improve the osteoblast adhesion. Biomaterials 2003; 24: 3663-3670.

33. van Delden CJ, Bezemer JM, Engbers GH, et al. Poly(ethylene oxide)-modified carboxylated polystyrene latices-immobilization chemistry and protein adsorption. J Biomater Sci Polym Ed 1996; 8: 251-268.

34. Shokri B, Firouzjah M and Hosseini SI. FTIR analysis of silicon dioxide thin film deposited by metal organic-based PECVD. In: Proceedings of 19th international symposium on plasma chemistry society, Bochum, Germany, 26-31 August 2009.

35. Ni H, Johnson AH, Soucek MD, et al. Polyurethane/ polysiloxane ceramer coatings: Evaluation of corrosion protection. Macromol Mater Eng 2002; 287: 470.

36. Lehle K, Buttstaedt $\mathrm{J}$ and Birnbaum DE. Expression of adhesion molecules and cytokines in vitro by endothelial cells seeded on various polymer surfaces coated with titaniumcarboxonitride. J Biomed Mater Res A 2003; 65: 393-401. 
37. Riescher S, Wehner D, Schmid T, et al. Titaniumcarboxonitride layer increased biocompatibility of medical polyetherurethanes. J Biomed Mater Res B Appl Biomater 2014; 102: 141-148.

38. Lehle K, Li J, Zimmermann HG, et al. In vitro endothelialization and platelet adhesion on titaniferous upgraded polyether and polycarbonate polyurethanes. Materials 2014; 7: 623-636.

39. Li X, Liu KL, Li J, et al. Synthesis, characterization, and morphology studies of biodegradable amphiphilic poly[(R)-3-hydroxybutyrate]-alt-poly(ethylene glycol) multiblock copolymers. Biomacromolecules 2006; 7 : 3112-3119.

40. Ko YG, Kim YH, Park KD, et al. Immobilization of poly(ethylene glycol) or its sulfonate onto polymer surfaces by ozone oxidation. Biomaterials 2001; 22: 2115-2123.

41. Anseth KS, Metters AT, Bryant SJ, et al. In situ forming degradable networks and their application in tissue engineering and drug delivery. J Control Release 2002; 78: 199-209.

42. Chen Z, Zhao M, Liu K, et al. Novel chitosan hydrogel formed by ethylene glycol chitosan, 1,6-diisocyanatohexan and polyethylene glycol-400 for tissue engineering scaffold: In vitro and in vivo evaluation. J Mater Sci Mater Med 2014; 25: 1903-1913.

43. Mahoney MJ and Anseth KS. Three-dimensional growth and function of neural tissue in degradable polyethylene glycol hydrogels. Biomaterials 2006; 27: 2265-2274.

44. Namba RM, Cole AA, Bjugstad KB, et al. Development of porous PEG hydrogels that enable efficient, uniform cell-seeding and permit early neural process extension. Acta Biomater 2009; 5: 1884-1897.

45. Lampe KJ, Bjugstad KB and Mahoney MJ. Impact of degradable macromer content in a PEG hydrogel on neural cell metabolic activity, redox state, proliferation, and differentiation. Tissue Eng Part $A$ 2010; 16: 1857-1866.

46. Shinohara S, Kihara T, Sakai S, et al. Fabrication of in vitro three-dimensional multilayered blood vessel model using human endothelial and smooth muscle cells and high-strength PEG hydrogel. J Biosci Bioeng 2013; 116: 231-234.

47. Lin L, Marchant RE, Zhu J, et al. Extracellular matrixmimetic poly(ethylene glycol) hydrogels engineered to regulate smooth muscle cell proliferation in 3-D. Acta Biomater 2014; 10: 5106-5115.

48. Asai T, Lee MH, Arrecubieta $\mathrm{C}$, et al. Cellular coating of the left ventricular assist device textured polyurethane membrane reduces adhesion of Staphylococcus aureus. J Thorac Cardiovasc Surg 2007; 133: 1147-1153.

49. Wiegand C, Bauer M, Hipler UC, et al. Poly(ethyleneimines) in dermal applications: Biocompatibility and antimicrobial effects. Int $J$ Pharm 2013; 456: 165-174.

50. Liu L, Gou M, Yi T, et al. Antitumor effects of heparinpolyethyleneimine nanogels delivering claudin-3-targeted short hairpin RNA combined with low-dose cisplatin on ovarian cancer. Oncol Rep 2014; 31: 1623-1628.

51. Jeon O, Yang HS, Lee TJ, et al. Heparin-conjugated polyethylenimine for gene delivery. $J$ Control Release 2008; 132: 236-242.

52. Roald HE, Barstad RM, Bakken IJ, et al. Initial interactions of platelets and plasma proteins in flowing nonanticoagulated human blood with the artificial surfaces Dacron and PTFE. Blood Coagul Fibrinolysis 1994; 5: 355-363.

53. Chien S. Mechanotransduction and endothelial cell homeostasis: The wisdom of the cell. Am J Physiol Heart Circ Physiol 2007; 292: H1209-H1224.

54. Chien S. Effects of disturbed flow on endothelial cells. Ann Biomed Eng 2008; 36: 554-562.

55. Robotti F, Franco D, Bänninger L, et al. The influence of surface micro-structure on endothelialization under supraphysiological wall shear stress. Biomaterials 2014; 35: 8479-8486.

56. Baier RE. Selected methods of investigation for bloodcontact surfaces. Ann NY Acad Sci 1987; 516: 68-77.

57. Yip J, Shen Y, Berndt MC, et al. Primary platelet adhesion receptors. IUBMB Life 2005; 57: 103-108.

58. Williamson MR, Black R and Kielty C. PCL-PU composite vascular scaffold production for vascular tissue engineering: Attachment, proliferation and bioactivity of human vascular endothelial cells. Biomaterials 2006; 27: 3608-3616.

59. de Mel A, Punshon G, Ramesh B, et al. In situ endothelialization potential of a biofunctionalised nanocomposite biomaterial-based small diameter bypass graft. Biomed Mater Eng 2009; 19: 317-331.

60. Diamantouros SE, Hurtado-Aquilar LG, Schmitz-Rode T, et al. Pulsatile perfusion bioreactor system for durability testing and compliance estimation of tissue engineered vascular grafts. Ann Biomed Eng 2013; 41: 1979-1989.

61. Achneck HE, Jamiolkowski RM, Jantzen AE, et al. The biocompatibility of titanium cardiovascular devices seeded with autologous blood-derived endothelial progenitor cells: EPC-seeded antithrombotic Ti implants. Biomaterials 2011; 32: 10-18. 